\title{
A UNIFIED N-BODY AND STATISTICAL TREATMENT OF STELLAR DYNAMICS
}

\author{
Alan P. Lightman \\ Harvard-Smithsonian Center for Astrophysics \\ and \\ Stephen L. W. McMillan \\ University of Illinois, Champaign-Urbana
}

\begin{abstract}
We summarize the methods of a new "hybrid" computer code for stellar dynamics. All particles in the inner spatial region are followed exactly via a direct $\mathrm{N}$-body code and all particles in the outer spatial region are treated statistically via a distribution function and Fokker-Planck type methods. An intermediate region, with features of both, allows exchange of particles and energy between the outer and inner regions. We apply our code to the period just before core collapse and just after and summarize the results.
\end{abstract}

\section{INTRODUCTION}

From theoretical work in stellar dynamics, we have known for forty years that the core of a self-gravitating system of point masses will collapse, with an ever-decreasing number of particles and an ever-increasing density. (See, e.g. Spitzer, 1985 for a review.) Such a collapse must eventually be halted by such processes as stellar collisions and binary activity. Recent comparisons of the collapse theory with observations of actual globular cluster cores suggest that some globular clusters have already collapsed and are now embarked upon post-collapse evolution (Lightman, 1982; Cohn and Hut, 1984). In the same recent period, theoretical investigations have focused on post-collapse

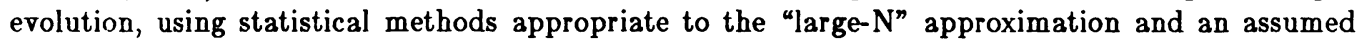
central heat source to drive the expansion (Stodolkiewicz, 1982; Goodman, 1984; Inagaki and LyndenBell, 1983; Heggie, 1984; Sugimoto and Bettwieser, 1984; see Heggie, 1985 for a review). Most of these studies indicate the post-collapse core expands, with an ever-increasing number of particles.

For an understanding of the evolution shortly before core collapse and shortly thereafter, the purely statistical methods are inadequate. This is because, for binaries the agent of collapse reversal and no initial binaries, the core will always collapse down to about 100 particles before reversal, independent of any other initial conditions (Lightman and Fall, 1978; Heggie, 1984). Statistical methods fail for such a small number of particles. On the other hand, the core is typically surrounded by a vastly larger number of particles, also evolving on the time scale of interest and requiring a statistical treatment. To allow study of the core during the critical period mentioned above, where both "small-N" effects and "large-N" effects are important, we have developed a hybrid computer code, in which particles are followed exactly in the inner spatial regions via a direct $\mathrm{N}$-body code and statistically in the outer spatial regions via a Fokker-Planck type code. In effect, our method provides a bridge between the purely statistical epochs of the pre- and post-collapse phases of evolution.

\section{SUMMARY OF THE HYBRID CODE}

\subsection{Physical Approximations}

The principal physical approximations are that all stars are treated as point masses, of initially equal mass, and binaries are initially absent. These approximations reduce the problem to one of pure stellar dynamics, with no essential free parameters. 


\subsection{Radial Zones and Methodology}

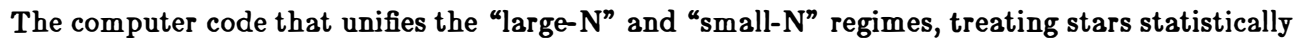
in some places but following orbits directly in others, is a "hybrid", combining many of the features of previous codes. The system is first divided into three fixed radial zones:

(i) an "N-body region", referred to below as "NB", defined by $r \leq r_{N}$,

(ii) an "inner Fokker-Planck region" ("FP1"), having $r_{N}<r \leq K r_{N}$, and

(iii) an "outer Fokker-Planck region" ("FP2"), with $r>K r_{N}$.

The radius $r_{N}$ is arbitrary, constant in time, and chosen as the unit of length in all that follows. K is a constant and $N$ is the number of stars within $r_{N}$ at any given time. The choices of $K$ and an initial value for $N$ are governed mainly by economic considerations; typical values are $K \sim 3, N \sim 100$ 150. Progressing outward from the center of the system, the three zones represent increasing degrees of approximation, as described below.

Given the three radial zones, a number of regions in energy and angular momentum space (E-J) space may be defined, according to the pericenter $\left(r_{-}\right)$and apocenter $\left(r_{+}\right)$of the corresponding orbits:

$$
\begin{aligned}
\text { I : } & r_{+}(E, J) \leq r_{N} \\
\text { II }: & r_{+}(E, J)>r_{N}, r_{-}(E, J)<r_{N} \\
\text { III } & r_{-}(E, J)>r_{N} .
\end{aligned}
$$

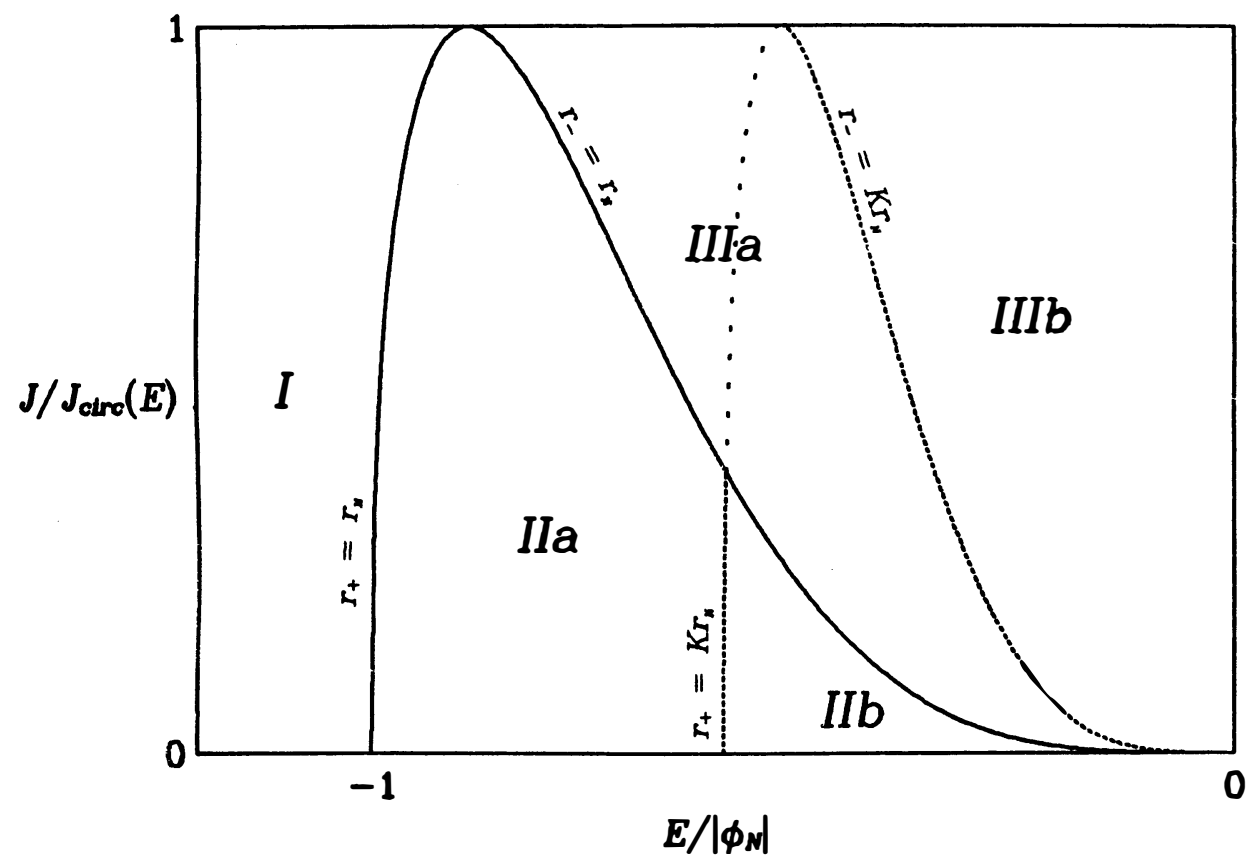

Figure 1: Regions of E-J space corresponding to different types of orbits.

\section{Region II}

it will be convenient to denote that part of region III with $r_{-} \leq K r_{N}$ as IIIa 
that region as IIIb. All these regions are shown schematically in figure 1 . The methods used to treat a star's orbit depend on both the radial zone and the phase-space region under consideration.

(a) Stars inside $r_{N}$.

Within NB, all stars are followed exactly using a three-dimensional N-body code. Since the presence of hard binaries is of such critical importance to the late evolution of isolated small-N systems, it is anticipated that it might be similarly important here also. For this reason, the force law is taken to be a simple inverse square - no softening of the potential is introduced, so hard binaries, should they occur, are correctly followed.

The program actually used is a somewhat modified fifth-order predictor-corrector procedure of the type developed by Aarseth (1968, 1974), employing individual timesteps and including a two-body Kustaanheimo-Stiefel (1965) regularization procedure, enabling simultaneous "close" encounters between up to twenty pairs of stars to be treated efficiently. The most major modification is, of course, that only stars within a fixed radius are considered. When a star crosses $r_{N}$ moving outward, it is removed from the $\mathrm{N}$-body integration, as follows. First, all stars within $r_{N}$ are integrated up to the current time. The escaper's contribution to the force and first three force derivatives felt by all other bodies in NB may then be calculated and subtracted out; its contribution to the binding energy is similarly removed (enabling energy conservation to be conveniently checked at any later time). Once this has been done, the $\mathrm{N}$-body system has "forgotten" about the existence of the star and the integration can proceed. When it becomes necessary to insert incoming stars, a similar procedure is followed; because of the pairwise nature of the expression for the force and higher derivatives, all of these quantities may be determined for the newcomer simultaneously with the application of the corrections to the rest of the system, with no extra computational effort. The treatment of stars in the outer two zones (see below) means that no additional special measures need be taken to account for their interaction with a star crossing $r_{N}$.

In the runs described below, the N-body part of the code ran on an FPS-120B Array Processor. This device, while considerably faster than the VAX 11/780 used for the remainder of the program, suffered from quite severe limitations on the amount of memory available.

(b) Stars with $r_{N}<r<K r_{N}$.

In the inner Fokker-Planck region, the trajectories of all stars are integrated in one dimension only. As a star enters this region, it is replaced by a spherical shell with the same mass and velocity, and the motions of all such shells are followed using a simple second-order integration scheme. The radial force per unit mass experienced by a shell of mass $m_{i}$, radius $r_{i}$ and angular momentum $J_{i}$ per unit mass is

$$
a_{i}=-\frac{M_{N B}+\frac{1}{2} m_{i}}{r_{i}^{2}}+\sum_{\substack{\text { ohelloj: } r_{j}: r_{j}<r_{i} \\ r_{j}}} \frac{m_{j}}{r_{j}^{2}}
$$

and the radial equation of motion is

$$
\ddot{r}_{i}=a_{i}+\frac{J_{i}^{2}}{r_{i}^{3}}
$$

Here, $\mathrm{M}_{N B}$ is the total mass of all the stars in the $\mathrm{N}$-body region (equal to $N m_{*}$ in the present case), regarded as located at $r=0$. The extra $\frac{1}{2} m_{i}$ in the first term takes into account the self-gravity of a shell.

Forcing spherical symmetry on the potential greatly simplifies the integration. However, information on the original star's trajectory is not discarded. Its angular coordinates are also known, since the plane of the orbit is known and the coordinate in that plane, $\theta$, satisfies 


$$
\dot{\theta}=\frac{J}{r^{2}}
$$

enabling full three-dimensional position and velocity information to be retrieved at any time (in particular, when the shell must be inserted into NB). The term "shell" should not be taken to mean that the object under consideration here represents more than one star, as it does in the work of Spitzer and coworkers (e.g. Spitzer and Hart, 1971). Rather, it simply reflects the fact that, dynamically, it reacts and contributes only to a spherically smoothed mass distribution and thus, for the purposes of the one-dimensional integration, behaves as though it were indeed a shell of material, albeit one with "hidden" parameters, such as orbital orientation, etc.

The one-dimensional force calculation described above allows shells to react to changes in their bulk distribution. It does not, however, permit the two-body effects that drive the evolution over long timescales. The two-body perturbation spectrum experienced by a shell can be generated in a manner similar to that described by Shapiro and Marchant (1978). The expected values of the orbital perturbations are obtained by integrating the local energy and angular momentum diffusion coefficients along a segment of the unperturbed orbit,

$$
\langle Q\rangle\left(r_{1}, r_{2}\right)=\int_{r_{1}}^{r_{2}} \frac{d r}{v_{r}(E, X ; \phi(r))}\langle Q\rangle_{t}
$$

for $Q=\Delta E,(\Delta E)^{2}$, etc. Here we have made the substitution $X=J^{2}$; and $v_{r}$ and $\phi(r)$ denote the radial component of velocity and gravitational potential at radius $r$, respectively. The actual orbital perturbation is then generated by randomly selecting $(\delta E, \delta X)$ from a two-dimensional Gaussian distribution having means $\langle\Delta E\rangle$ and $\langle\Delta X\rangle$ and second moments $\left\langle(\Delta E)^{2}\right\rangle,\left\langle(\Delta X)^{2}\right\rangle$ and $\langle\Delta E \Delta X\rangle$. The component integrals are accumulated as the shell's orbit is calculated and the random perturbation is applied when any of a number of criteria is met:

(i) one of the perturbations, $\langle\Delta E\rangle^{2},\left\langle(\Delta E)^{2}\right\rangle,\langle\Delta X\rangle^{2},\left\langle(\Delta X)^{2}\right\rangle$, becomes unacceptably large, or

(ii) the shell is about to leave FP1, either at $r_{N}$ or at $K r_{N}$, or

(iii) the shell passes apocenter in FP1. In this case, the shell is then relocated to the apocenter of its new orbit and its acceleration (and that of all other shells in the system) is corrected before the integration continues.

Because the determination of the phase-space distribution function and the subsequent integration over energy for every shell at every timestep would be prohibitively expensive, to compute the diffusion coefficients we assume that the distribution is Mawellian at low energy and power-law at high energies, as given by the "large- $\mathrm{N}^{\text {" calculations. }}$

Because of their potential dynamical importance, it is essential that hard binaries not be "lost" as they cross $r_{N}$ moving outward. Thus, when the center of mass of such a pair leaves NB, it is replaced by a shell of mass $2 \mathrm{~m}$. However, in addition to all the usual parameters (angular momentum, orbital orientation, label, etc.) associated with a shell, the pair's binding energy at removal is also stored. Pcrturbations to the shell's orbit are determined and applied as described above, with the following two changes. First, the additional mass of the binary is taken itno account when applying the diffusion coefficients. Second, as shown by Heggie (1975), the binding energy, B, of a hard binary moving through a field of stars with density $n$ and velocity dispersion $\left\langle v^{2}\right\rangle$ increases at a rate

$$
\dot{B} \approx 10 G^{2} m_{*}^{2} n\left\langle v^{2}\right\rangle^{-1 / 2},
$$

so, in addition to the perturbations to the orbital parameters, the binding energy is increased by the (usually small) amount

$$
\delta B=\int_{r_{1}}^{r_{2}} \frac{d r}{v_{r}} \dot{B}
$$

whenever a perturbation is applied. 
No attempt is made to follow either the orientation or the eccentricity of the relative orbit of the pair. The transit time of the center-of-mass shell through FP is likely to be many binary orbital periods, so it is simply assumed that both quantities are random at reinsertion, with the eccentricity $e$ having a distribution $\Phi(e)=2 e$ (Jeans, 1929). Reinsertion of the pair is accomplished by choosing some point on the relative orbit, inserting the two components separately and allowing the $\mathrm{N}$-body code to perform the necessary initialization and regularization. It should be emphasized that only hard binaries are treated in this way. Pairs emerging from NB with $B<\frac{1}{2}\left\langle v^{2}\right\rangle$ are broken up and their components treated separately.

\section{(c) Stars outside $K r_{N}$}

Following the general approach laid down earlier, stars in FP2 are followed in $(E, X)$ space only. Furthermore, and in contrast to the treatment in FP1, the potential and background distribution function used to perturb the orbits is FP2 is held fixed. This approximation, which we hope to eliminate in future work, must lose its validity on a time of order the relaxation time at $K r_{N}$.

Whether the potential in FP2 is held fixed or allowed to vary, the diffusion and perturbations of stars in FP2 is treated as follows: The IIIb stars, those that never come within $K r_{N}$, may be treated using any of the large- $N$ techniques mentioned earlier - the Cohn (1979) and ShapiroMarchant (1978) methods are most appropriate for the configuration used here. Accordingly, region IIIb is covered with a grid or realized in the form of "superstars" and the evolution is followed by considering fluxes of stars between neighboring grid cells or random perturbations to the superstars' orbital parameters, generated much as described above. In general, one would expect the relaxation time at large radii to be much greater than that in the central regions, so very few FP2 timesteps need be taken during a typical run covering many core relaxation times.

This outermost portion of the calculation communicates with the shell integration via the IIb and Illa stars that are "in transit" through FP2. Given the potential outside $K r_{N}$, the orbits, and the perturbations applied to the orbits, of shells outside $K r_{N}$ can be calculated. When an outwardmoving shell crosses $K r_{N}$, it is perturbed by an amount calculated, as before, from the moments

$$
\langle\Delta E\rangle_{K}=2 \int_{K r_{N}}^{r_{+}} \frac{d r}{v_{r}}\langle\Delta E\rangle_{t},
$$

etc., and "absorbed" into the IIb or III distribution in FP2. As mentioned above, no orbital phase or orientation information is retained. Instead, region IIb/IIla in phase-space is divided into 1500 bins ( 100 in $\mathrm{E}, 15$ in $\mathrm{X})$. Associated with each bin are three numbers: the number of stars it contains, $\mathcal{N}$, the mean rate, $R$, at which stars from that bin are to be inserted into FP1 and the time of the next insertion. On insertion, the shells "hidden" orbital orientation is generated randomly. When a shell is added to or removed from a bin, the occupation number and insertion rate are adjusted accordingly. This procedure has the desirable property that it allows the bin occupation number to react rapidly to fluctuations in the rate at which shells arrive from FP1; the reinsertion rate changes on a timescale $R^{-1}$ - less than the return time for an individual shell by a factor of order $N^{-1}-$ thus tending to smooth the irregularities out. The (extremely) rare event of a binary shell leaving FP1 is treated by attaching a "flag" to the bin in question indicating the binding energy and the number of insertions which must occur before the binary returns.

\subsection{Summary of Error Corrections}

The method used to integrate the shell equations of motion, being only of second order, is inherently much less accurate than the N-body integration. However, the energy error can be calculated at each timestep at no extra computational cost and a correction can be applied. The underlying reason for the reduced accuracy is the fact that shells can cross and, when they do, the force experienced by each of the crossing pairs changes discontinuously. The neglect of shell crossings 
would cause the energy to increase secularly. By keeping track of the relative ranks of shells before and after each time step, the net number of crossings for each shell can be determined and an energy correction applied.

Another source of accumulated error stems from the fact that a finite number of perturbations drawn from a given distribution will generally not have the same mean as the distribution itself. To avoid undesirable spurious fluxes of energy and angular momentum from one part of phase space to another, care must be taken to ensure that, over a period of a few crossing times, the desired perturbation distribution is correctly generated. Accordingly, regions II and IIla are divided into 100 cells on a $10 \times 10$ grid. As each perturbation is applied, the actual perturbations, $(\delta E, \delta X)$, the calculated mean perturbations, $(\langle\Delta E\rangle,\langle\Delta X\rangle)$, and the identity of the shell perturbed are recorded against the appropriate cell. Once a sufficient number (chosen to be 15) of perturbations has accumulated in a given cell to ensure adequate statistics, the energies and angular momenta of the stars in question are adjusted to force the averages of the actual perturbations to the desired mean values. It is assumed that no additional correction need be applied to force the second moments of the distribution in a similar way, although such a correction could be applied if necessary.

\subsection{Tests of the Code}

We performed a number of tests on our code, checking energy conservation and other such desireable properties. One test, described here, was the approach to thermal equilibrium under appropriate conditions. As shown by Antonov (1962) and by Lynden-Bell and Wood (1968), a collection of $N$ point masses interacting only gravitationally and confined within a sphere will attain and maintain thermal equilibrium provided certain conditions are satisfied: (1) binary interactions must be ignored; (2) if $E$ and $M$ are the total energy and mass, respectively, of the confined particles and $R_{\theta}$ is the radius of the spherical shell confining the particles, then $E$ must exceed the value -0.335 $G M^{2} / R_{o}$; and (3) the central density must be less than 709 times the density at $R_{e}$.

Our test consisted of placing a reflecting shell at $R_{\bullet}=K r_{N}$, with $\mathrm{K}=3$, introducing an effective cutoff (or "softening parameter") in the short range interaction of particles to prevent hard binary formation, and beginning the system far from thermal equilibrium. In particular, we initialized the system with 300 particles in NB, at constant density and with flat velocity distribution, and no shells

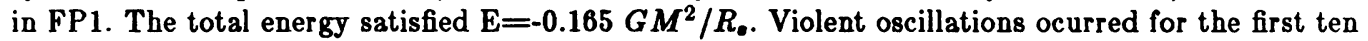
crossing times, after which the system began to relax toward a thermal distribution. After about thirty central relaxation times, the mean velocity dispersion leveled off at its equipartition value and, by sixty central relaxation times, stochastically departed from this value by only about $4 \%$. Moreorer, the velocity dispersions of both the particles in NB and the shells in FP1 were essentially identical after thirty central relaxation times, at which time approximately 200 of the initial particles were in the form of shells. Both the density profile and the velocity distribution approached those of an isothermal distribution appropriate to the fixed parameters of the system, with the one exception that, at about sixty central relaxation times (the end of the run), the high velocity tail of the distribution (with squared velocities larger than 2.5 times the mean) had approximately $25 \%$ too much kinetic energy. Because of the steep increase of relaxation time with velocity, these particles and shells had not yet had time to relax.

This test proved extremely useful as a diagnostic tool. Errors in the algorithms used in the generation and correction of perturbations and the redistribution of the errors incurred at $r_{N}$ invariably showed up as secular trends in the core parameters and/or the heating or cooling of some radial zone or zones. Furthermore, the similar and correct behavior of both shells and particles suggests that no important error is being introduced by the artificial boundary at $r_{N}$ and the different methods of treatment inside and outside of this boundary. 


\section{APPLICATIONS AND RESULTS FOR CORE BOUNCE}

\subsection{Initialization}

In the most recent calculations of core collapse, the Fokker-Planck treatment of Cohn (1980) and the fluid treatment of Lynden-Bell and Eggleton (1980), the late stages of collapse become homologous and have the solution

$$
\begin{gathered}
n(r, t) \propto r^{-\alpha}, r \gg R_{c}(t) \\
R_{c}(t) \equiv\left(\frac{3\left\langle v^{2}\right\rangle_{c}}{4 \pi G m_{\bullet} n_{c}}\right)^{1 / 2} \propto\left(1-\frac{t}{t_{c o l l}}\right)^{2 /(6-\alpha)} \\
n_{c}(t) \equiv n(o, t) \propto R_{c}^{-\alpha} \\
\left\langle v^{2}\right\rangle_{c} \equiv\left\langle v^{2}\right\rangle(o, t)=2 G m \cdot N_{c} R_{c}^{-1} \propto N_{c}^{(2-\alpha) /(3-\alpha)} .
\end{gathered}
$$

Here time $t$ is measured from the beginning of the homologous phase, $r$ is the radial distance from the center of the (spherically symmetric) system, $\mathrm{n}$ is the density, $R_{c}$ the core radius, $m$. the mass of a typical star in the core, $\left\langle v^{2}\right\rangle$ the squared velocity dispersion, $N_{c}=(2 \pi / 3) n_{c} R_{c}^{3}$ the number of stars in the core, $\alpha=2.23$, and $t_{\text {coll }}$, the collapse time, is $200-300$ initial central relaxation times. Subscripts c on all quantitites will denote central (core) values. The equality in Equation (6d) expresses the virial relationship. In the region $r \lesssim R_{c}$, the distribution is that of an isothermal sphere, with an approximately constant density. Henceforth, we shall refer to the above solution as the CL-BE distribution.

Ideally, we would like to initialize our system with a configuration corresponding to a snapshot of the CL-BE distribution, at a late stage of the collapse, but not so late that the statistical approximation has yet broken down. This would involve at least several hundred particles in the core and an $\mathrm{N}$-body region capable of following a similar number of particles. For practical reasons, we have been limited to an initial value of the number of particles in the $\mathrm{N}$-body region, $\mathrm{N}$, equal to 100. ( $\mathrm{N}$ changes in time; in particular, it increases as the collapse proceeds.) For an initial value of $\boldsymbol{R}_{c} / \boldsymbol{r}_{N}$ we take 0.6 . We then choose a random distribution of $\mathrm{N}$-body particles and Fokker-Planck shells representing the CL-BE distribution with these values of $N$ and $R_{c} / r_{N}$. Initially, the number of particles in the core, $N_{c}$, is 55 and the number of shells in FP1 is 220. All times will be measured in units of the time-independent quantity $t_{N}$, defined by

$$
t_{N} \equiv \frac{r_{N}}{\left|\phi_{N}\right|^{1 / 2}} \quad \text { at } \quad t=0
$$

where $\phi_{N}$ is the central potential. The time scale $t_{N}$ is approximately a quarter of the initial Nbody-region crossing time. With our assumed initial parameters and distribution, the core relaxation time

$$
t_{R c} \equiv \frac{\left\langle v^{2}\right\rangle_{c}^{3 / 2}}{15.4 G^{2} m_{*}^{2} n_{c} \ln \Lambda}
$$

intitially satisfies $t_{R c}=6 t_{N}$. Here $\ln \Lambda \approx \ln 0.4 N_{c}$ is the gravitational Coulomb logarithm.

Since stars are treated as point particles, there is no overall length or time scale imposed upon the problem, except by reference to observed globular clusters and the number of particles in the core. With the scaling laws given by the CL-BE distribution and characteristic values $N_{c} \sim 10^{4}$ and $R_{c} \sim 1 \mathrm{pc}$ for a globular cluster at the time it begins its homologous collapse, we may attach the following physical dimensions to the core at the time it has reached $N_{c} \approx 55: r_{N} \sim 2 \times 10^{-3}$ pc, $t_{N} \sim 9$ yrs., $t_{R c} \sim 50$ yrs. 


\subsection{Overall Behavior}

The overall behavior of the system can be seen in Figure 2. This shows the time-dependence of two measures of the central density. The solid line shows the mean density within $0.25 r_{N}$ of the geometric center of the system (defined at large radii), and the dotted line shows the mean density within the core using Von Hoerner's (1963) definition of the core radius.

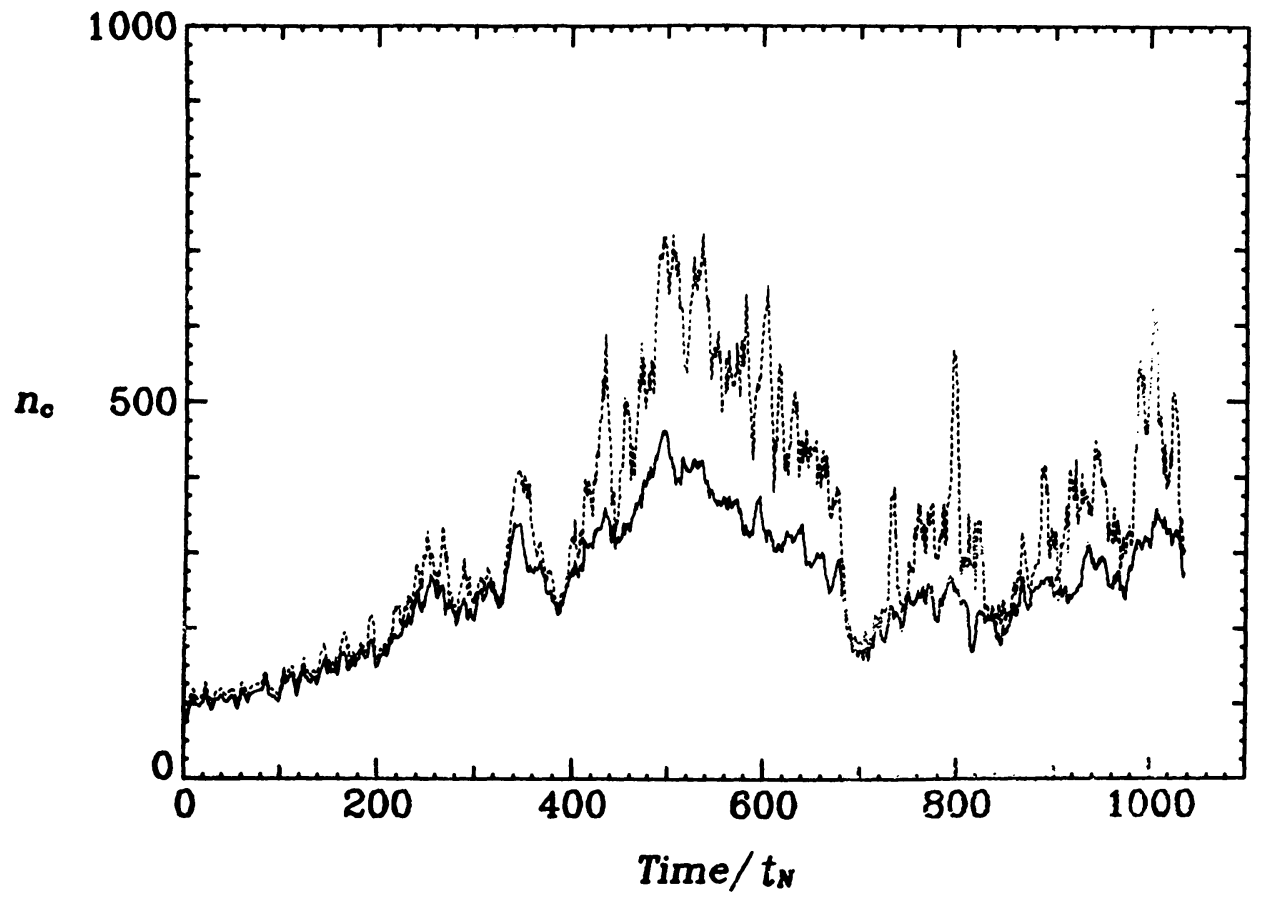

Figure 2: Central density as a function of time.

The evolution may be conveniently divided into a number of distinct phases:

(A) The initial collapse phase, from $t=0$ to $t \approx 500 t_{N}$. Here, the system continues to collapse along the "large $-N^{\prime}$ track, following the CLBE solution, until the number of stars in the core becomes very small ( $\sim$ 25) and the contraction is halted by the formation and hardening of a hard binary.

(B) The hard binary continues to harden, driving an expansion phase for $500 t_{N} \lesssim t \lesssim 680 t_{N}$. This phase continues until the binary is ejected from the core by a violent triple encounter.

(C) The recollapse phase, $t \gtrsim 680 t_{N}$, in which, the internal heat source removed, the core begins to collapse again. Once the central density increases sufficiently, new binaries form and begin to harden and, by the end of the run, are injecting energy into the central regions at a rate almost sufficient to start a second expansion. 


\subsection{Homologous Initial Collapse Phase}

The projected core collapse time is best obtained by fitting a straight line to the decreasing central relaxation time as a function of time (any homologous collapse should show such a linear behavior), giving a collapse time of 110 initial central relaxation timescales. See Figure 3 . We suggest that this is somewhat shorter than the values of 200-300 found by Cohn $(1979,1980)$ because of difficulty in balancing the diffusion coefficients in the Fokker-Planck region with the actual encounters in the $\mathrm{N}$-body region. In addition, deviations from isothermality will be higher in any stochastic realization with a small number of particles, and this speeds up the evolution.

The behavior of the "central" parameters $R_{c}, n_{c},\left\langle v^{2}\right\rangle_{c}$, again with the two definitions of the term "central" described above, are displayed in Figure 4, for the initial phase. Using the collapse time determined above, these parameters are fitted with the CL-BE curves and the fit is extremely good. Further evidence for the homology of the collapse is given in Figure 5, which shows that the density profile follows a one-parameter family throughout this phase. It is miraculous that the homology continues long after the statistical approximation has broken down.

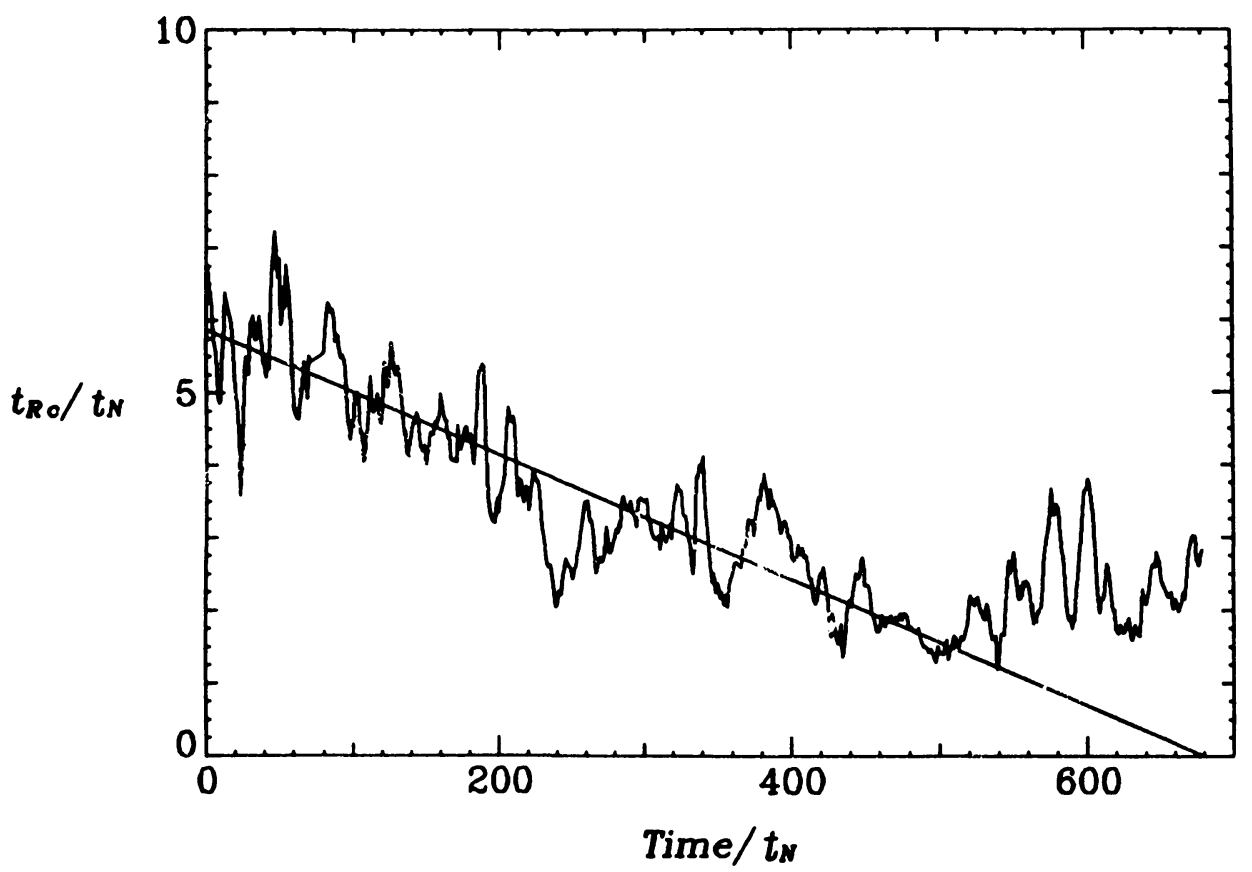

Figure 3: Central relaxation time as a function of time. 

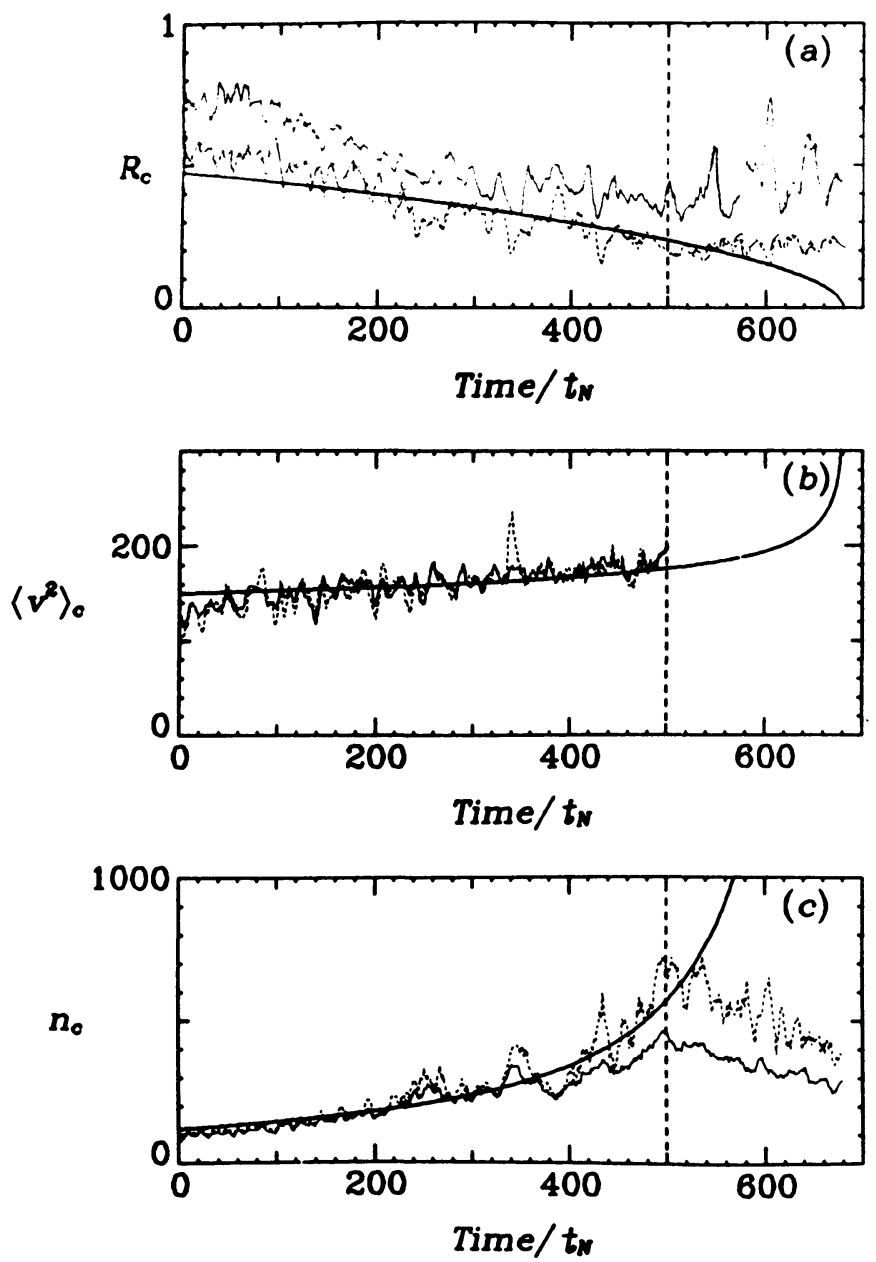

Figure 4: Core radius (a), central velocity dispersion (b), and central density (c) as a functions of time. The solid curves the predictions of the "large- $\mathrm{N}$ " theory of collapse. 


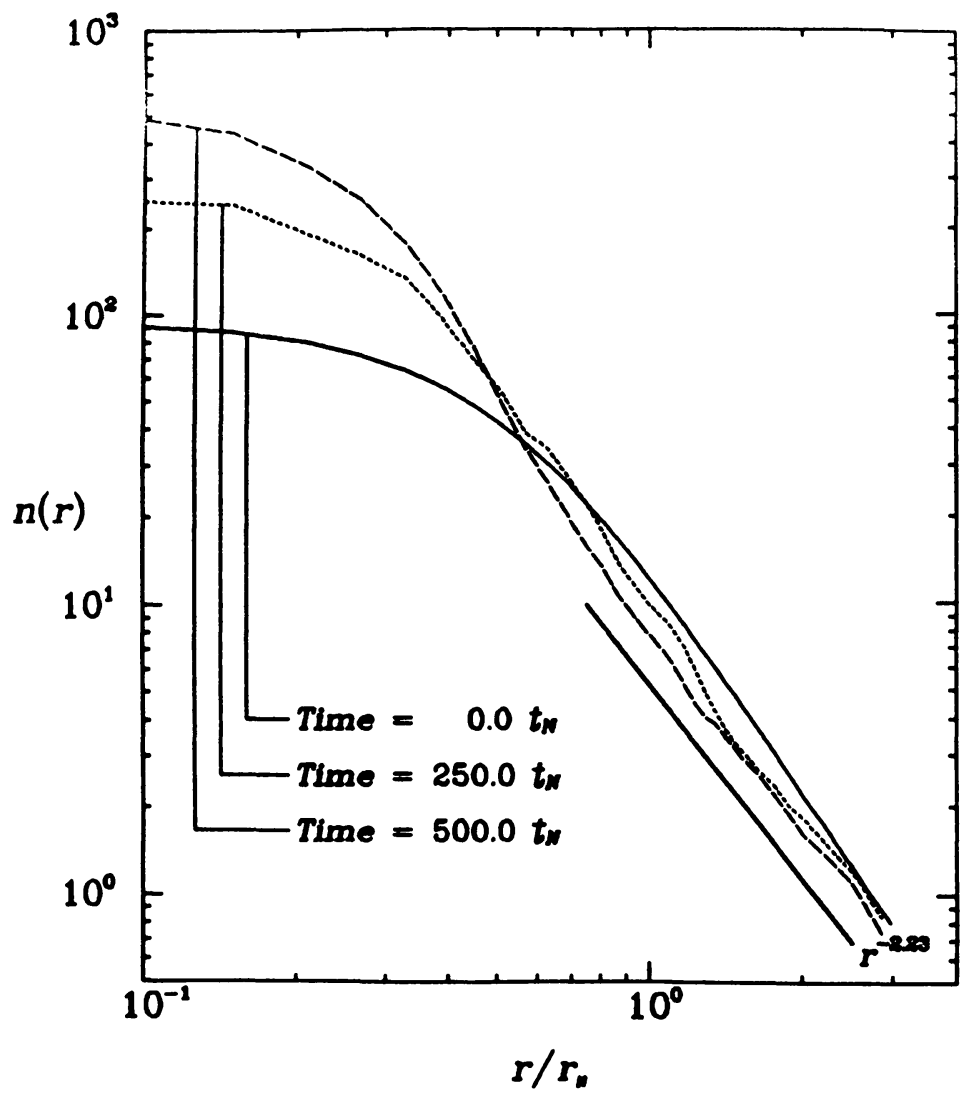

Figure 5: Density profile at three different times, ending at core collapse.

\subsection{Expansion and Recollapse}

The expansion phase was driven by the formation and heat input of a central binary as can be seen in Figure 6. As soon as the binary formed and began to harden, the core began to expand. The overall energetics of the expansion can be understood by simply noting that, at the time of maximum density, $N_{c} \approx 25$ and $\left\langle v^{2}\right\rangle_{c} \sim 180 \mathrm{Gm} \cdot / r_{N}, 80$ the total core kinetic energy is $T_{c} \sim$ $2250 \mathrm{Gm}_{*}^{2} / r_{N}$. The total change in the binding energy of the pair between $t=500 t_{N}$ and $t=600 t_{N}$ was approximately $3000 \mathrm{Gm}_{*}^{2} / r_{N}$, so, even allowing for considerable heating inefficiency, the energy input was sufficient to cause a substantial change in the core parameters. During the expansion of the core, the density profile followed the same one-parameter sequence of shapes as in the initial collapse.

At $t \approx 625 t_{N}$, the hard binary formed a relatively tightly bound $(H \sim 1)$ triple system with a third star. Here $H$ is the binary binding energy divided by the mean kinetic energy of a star. The system was hierarchical in nature, that is, the semi-major axis of the binary-single star orbit was initially much greater than the separation of the members of the binary, although the outer orbit rapidly began to harden and had reached $H \sim 2.5$ by the time special steps were taken to deal with it. 


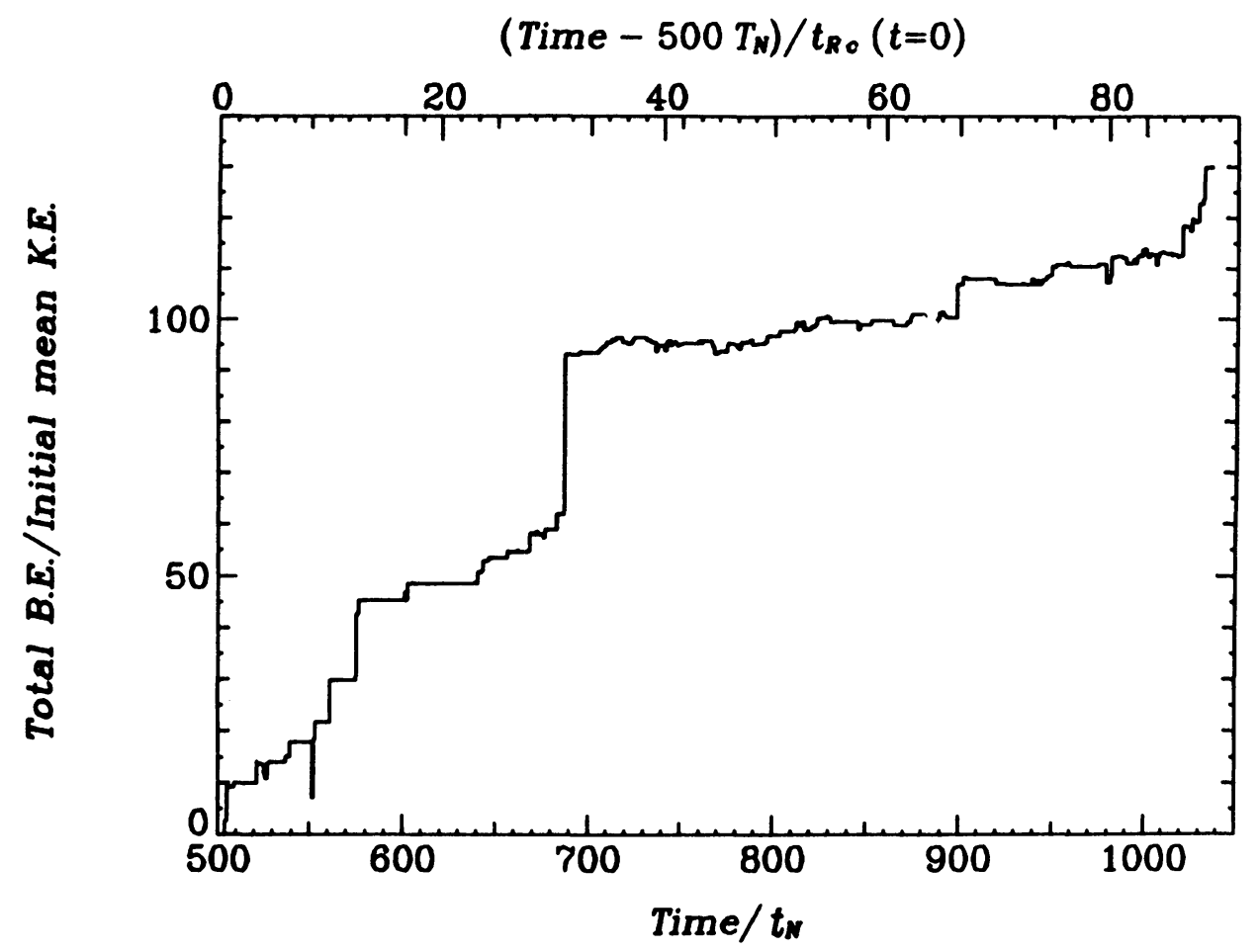

Figure 6: Binding energy in binaries as a function of time.

To enable the integration to proceed, we collapsed the inner hard binary to a single particle of double mass while the outer particle was becoming increasingly bound from encounters with field particles. Eventually, the shrinking orbit of the outer particle forced an end to the approximation. Once a triple loses its hierarchical character, there is strong numerical evidence (Agekian and Anosova, 1968; Harrington, 1972; Szebehely, 1972) that it becomes unstable. Harrington finds empirically that this happens when the pericenter of the outer orbit is a few times the scale of the inner one. Apportioning the recoil energy among the decay products gave the binary center of mass a velocity of 1.8 times the root mean square core velocity, so we simply ejected the binary from the system at this point.

At $t \approx 680 t_{N}$, with the central binary gone, the core began recollapsing. During the remainder of the run three more binaries formed and, near the end, two of them were hardening at a rate sufficient to begin a new expansion.

\section{SUMMARY OF RESULTS AND CONCLUSIONS}

\subsection{Summary of Results}

In brief, our results are as follows: (1) The homologous collapse continues into the "small-N" regime, all the way until the core bounces. (2) After the first core collapse, the core undergoes a series of expansions and contractions, forming a couple of new binaries in each cycle. Eventually, binary-binary interactions must become important. We believe this behavior is inevitable, given the small number of particles in the core at the time of core bounce. (3) The density profile is unchanged in the outer regions, at several core radii and beyond, and follows a 1-parameter regular isothermal form in the inner regions. 


\subsection{The Long Term Post-Collapse Evolution}

The long-term evolution of the system in post-collapse expansion has been recently studied in the fluid approximation by a number of investigators. Heggie (1984) finds that the inner regions form a regular isothermal sphere, out to a radius $R_{*}(t) \propto\left(t-t_{\text {coll }}\right)^{2 /(6-\alpha)}$, at least until this radius expands to the half-mass radius of the system. The density at $R_{*}$ has the form $n_{*} \propto R_{*}^{-\alpha}$. The above results can be quantified completely with the additional relation $t_{R}\left(R_{*}\right) \approx\left(t-t_{\text {coll }}\right)$ at all times during the expansion. The core quantities obey the relations $n_{c} \propto n^{(7 \alpha-12) / 3 \alpha}$ and $R_{c} / R_{*} \approx$ $\left(n_{c} / n_{*}\right)^{-1 / 2} \propto n^{(6-2 \alpha) / 3 \alpha}$. The outward luminosity necessary to maintaia this expansion, at the radius $R_{*}$, satisfies $L\left(t, R_{*}\right) \equiv L_{*}=0.4 n_{*} R_{*}\left(v^{2}\right)_{*}^{1 / 2} G m_{*}^{2}\left(\frac{300}{\gamma}\right) \ln \Lambda$, where $\gamma$ is the number of central relaxation times to collapse.

The dashed line in Figure 6 gives the integral of the above luminosity expression, assuming all of this energy is provided by binaries. The shape is not in bad agreement with our numerical results and the overall amplitude is also in agreement to within a factor of two.

If the long-term fluid calculations are valid, we can predict that the core oscillations observed shortly after core bounce will eventually damp out. Core oscillations are driven by the discrete formation and ejection of single binaries in the core. When the time to form a new binary, $t_{b i n}$, is much shorter than the core evolution time scale, $\tau_{c}$, we expect the oscillations to cease - the core as a whole then responds to a time-averaged binary heat input, just as does the halo, and a steady-state of secular core expansion is possible. The time scale for forming a new binary, ignoring the logarithmic term, satisfies $t_{b i n} \sim N_{c} t_{R c}$. The core evolution time scale can be defined as $\tau_{c}=N_{c}\left\langle v^{2}\right\rangle_{c} / L$. (a definition that keeps $\tau_{c} \propto t_{\text {coll }}$ during the collapse phase, when $R_{*} \propto R_{c}$ ). Finally, we write everything in terms of $R_{*}$, using the results $\left\langle v^{2}\right\rangle_{*} \approx\left\langle v^{2}\right\rangle_{c}$, the virial theorem $\left\langle v^{2}\right\rangle_{*} \propto n_{*} R_{*}^{2}$, n. $\propto R_{*}^{-\alpha}$, we obtain

$$
\tau_{c} / t_{b i n} \propto R_{*}^{\frac{3-a}{8}} \propto R_{*}^{0.28} \text {. }
$$

As $R$, increases with time in the post-collapse evolution, $t_{\text {bin }}$ monotonically decreases relative to $\tau_{c}$. Putting in the numerical factors, it is straightforward to show that this ratio is of order unity at the moment of the initial core collapse, when the oscillations are observed in the hybrid code. We thus expect these ocillations to cease after $R_{*}$ has increased by a factor of a few.

In conclusion, discrete $\mathrm{N}$-effects are quite visible in our calculations, but absent in the "large- $\mathrm{N}^{\text {" }}$ fluid approximations. Our results are consistent with the "large-N" post collapse evolution, but do not extend far enough in time to confirm them. If a constant density, secularly expanding core is confirmed, theory and observations of globular clusters may be brought into agreement.

This work was supported in part by NASA grant NAGW-246.

\section{REFERENCES}

Aarseth, S. J.: 1968, Bull. Astron., 3, p. 105.

Aarseth, S. J.: 1974 Astron. Astrophys., 35, p. 237.

Agekian, T. A. and Anosova, Zh. P.: 1968, Astrophys., 4 , p. 11.

Antonov, V. A.: 1962, Vestn. Leningr. Gros. Univ., 7 , p. 135.

Cohn, H.: 1979, Ap. J., 234, p. 1036.

Cohn, H.: 1980, Ap. J., 242 , p. 765.

Goodman, J.: 1984, Ap. J., 280, in press.

Harrington, R. S.: 1972, Celest. Mech., 6, p. 322.

Heggie, D. C.: 1975, M. N. R. A. S., 178, p. 729.

Heggie, D. C.: 1984, M. N. R. A. S., 206, p. 179.

Heggie, D.C.: 1985, this volume.

Hénon, M.: 1964, Ann. Astrophys., 27, p. 83. 
Hoerner, S. von: 1963, Z. Astrophys., 57, p. 47.

Inagaki, S. and Lynden-Bell, D.: 1983, M. N. R. A. S., 205, p. 913.

Kustaanheimo, P. and Steifel, E. J.: 1965, J. Math., 218 , p. 204.

Lightman, A. P.: 1982, Ap. J. Lett., 263, p. L19.

Lightman, A. P. and Fall, S. M.: 1978, Ap. J., 221, p. 567.

Lynden-Bell, D. and Wood, R.: 1968, M. N. R. A. S., 138, p. 495.

Lynden-Bell, D. and Eggleton, P. P.: 1980, M. N. R. A. S., 191, p. 483.

Shapiro, S. L. and Marchant, A. B.: 1978, Ap. J., 225, p. 603.

Spitzer, L. and Hart, M. H.: 1971, Ap. J., 164, p. 399.

Spitzer, L.: 1985, this volume.

Stodolkiewicz, J. S.: 1982, Acta Astron., 32, p. 63.

Sugimoto, D. and Bettwieser, R.: 1983, preprint.

Szebehely, V.: 1972a, A. J., 77, p. 169.

Szebehely, V.: 1972b, Celest. Mech., 6, p. 84. 